\title{
A Data Security Model for Altering Data Ecosystem and Affirmatively Pre- vent Mass Data Breaches
}

Jianqing Wu, Ph.D., J.D., Ping Zha, M.D.

Correspondent Author:

Jianqing Wu, Ph.D., J.D. tempaddr2@atozpatent.com.

Bios of the author: Jianqing Wu, Ph.D., J.D. has developed internet security technologies for nearly two decades with four granted U.S. Patents in data storage security. On another hat, he also has done pioneer medical researches to find cures for chronic diseases.

Abstract

This article discusses the root cause of prevalent mass data breaches and new approach to preventing mass data breaches. The prevalent mass data breaches can be attributed to the trusted business concept. In an internet system constructed on trusted relationship, consumer data records for all consumers can be cracked in the same way. Potential rewards from a successful hacking are much larger than the cost needed to crack the system. In such a system, whatever data security measures placed on the business's server can be defeated by insider's help which can be secured by sharing only a small part of rewards. A realist solution cannot be merely improving data security. Most business transactions between two parties can be conducted with minimum mutual trust and minimum personal data. A viable security measure must depart from the trusted business model but be designed to reach a sophisticated balance between trust and distrust. I will show that encrypting consumer data by the consumer's own keys in a semi-trust business transaction model will put an end to repeating mass data breaches. I will discuss the conditions for using such encryption technologies and how to address the big nightmare that more than half of U.S. residents might have lost their personal identity data. Finally, I will show how the new technologies can prevent a variety of damages to consumers, federal government, state governments, and societies (particularly most noninternet companies and insurance companies).

Data Security, (c) JWu-v100 


\section{INTRODUCTION}

Internet e-commerce system model has been influenced by trust business concept [1-3]. In order to conduct a business transaction between a vendor and a consumer, they must establish a trusted relationship. System trust is important to consumers and can be influenced directly by the vendor through provision of guarantees. Most early studies focused on buyer-and-sell transaction unrelated to e-commerce [3-4]. For an vendor to succeed, the vendor must win trust from consumers in the competitive world [5-12]. In order to maintain continuous business relationship, vendors need consumer information. To achieve the maximum convenience, it is desirable to have consumer data stored on the server so that the vendor can use them whenever they need data. The vendor takes personal information, address, banking information, credit card, etc. and keeps the data in the same way by using the same security measures. When the vendor becomes larger and larger, the magnitude of risk of leaking consumer data also increases. This results in endless mass data breaches. Wikipedia documented about 349 mass data breaches [13-14]. Most of small data breaches may never get attention and thus escape from scrutiny. The database of Privacy Right lists more than 9,000 data breaches [15]. Over the decades, it is hoped that data security can be achieved by improving user identity validation method. However, this line of data security measures work only marginally because they can be cracked for the same reason. However, the biggest risk is not from cracking user passwords through internet connection, but hacking the database storing millions of consumer records most probably with insider's help. One worst data breach with the MEGA a popular cloud service exposed 772,904,991 emails and 21,222,975 passwords [16]. In this study, I will show a workable security measure must depart from trusted system concept and change the practices of data broker industry in collecting and selling consumer data.

\section{FLAWS IN EXISTING DATA SECURITY MODEL}

\section{A. The History of Consumer Data Breaches}


Internet history reflects failure in protecting consumer data. The Wikipedia shows a list of 349 well known mass data breaches for those involving the theft or compromise of 30,000 or more records and those involving large corporations [13]. It was estimated that the total damage caused by data breaches is between $\$ 375$ and $\$ 575$ billion annually in the U.S. The global annual cost forecast is about $\$ 2.1$ trillion. Despite a large number of patent grants in the U.S. alone, there is no sign that existing technologies can ever stop mass data breaches. Many of those recent breaches were found with technical giant companies. This fact that frequent data breaches with technologies companies discredits the claim that technologies lone can solve the problem. Technology companies that have experienced mass data breaches include Microsoft (2019, 2021), HP (2006), AT\&T (2008, 2010), Adobe System (2013, 2019), Sony (2011), Dropbox (2012), Yahoo (2012, 2013, 2013, 2014), Google Gmail (2014, 2018), Tmobile (2015), Mango DB (2019, 2019), and Facebook (2013, 2018, 2019, 2019, 2019). Many companies experienced data breaches multiple times: Facebook (5), Yahoo (4), Sony (4), Google (2), U.S. army (2), and T-mobile (2). For the last year (2021), 13 mass data breaches were reported: Apple; Apple, Inc./BlueToad; Apple Health Medicaid; Microsoft Exchange servers; T-Mobile; NEC Networks, LLC; AOL; CyberServe; Atraf; Twitch; Health Service Executive; International Committee of the Red Cross. A large number of mass data breaches happened to banks, credit card companies, credit reporting agencies, health care systems, and schools and universities. Even federal agencies such as army, US homeland security, and US defense agencies were unable to safe keep their data. It should be understood that reported data breaches are only large ones that cannot escape from public scrutiny. Real number of data breaches would be much more. Based on the number and pattern of data breaches and the technical and financial strengths of the involved companies, I can reasonably conclude that it is impossible to stop mass data breaches by using security technologies consistent with current thinking.

According to the nonprofit consumer organization Privacy Rights Clearinghouse, a total of 227,052,199 individual records containing sensitive personal information were involved in security breaches in the United States between January 2005 and May 2008, excluding incidents where sensitive data was apparently not actually exposed [15]. In 2019, a collection of 2.7 billion identity records, consisting of 774 million unique email addresses and 21 million unique passwords, was posted on the web for sale [16]. The total number of consumer records exposed in 2021 is at least 375 million which is based on the best esti- 
mates (excluding those without providing the number of records). The accumulative data loss is extremely large.

The impacts of breached consumer data may be enhanced by unregulated access to data broker market [17]. Data broker is now more than $\$ 200$ billion industry [18]. A data broker collects consumers data from federal government (the U.S. Census Bureau), state agencies, retailers, other data brokers, and internet companies that use cookies and the third-party cookie [19-20]. One of the companies the FTC studied, Acxiom, boasted that "[i]ts databases contain information about 700 million consumers worldwide with over 3,000 data segments for nearly every U.S. consumer." [19]. If I combine the consumer data from mass data breaches and consumer data traded in the data broker industry, few U.S. residents can keep their identity data.

\section{B. Two Incentives for Data Breaches and Data Flow}

Consumers data may be sold for two different purposes: some data are sold as market leads and others sold for defrauding consumers, governments, and societies. The Social Security number of someone with good credit, for instance, can sell for between $\$ 60$ and $\$ 80$ [20]. For example, the list sale prices for the Brazilian underground: a set of business application account credentials \$155193 , a set of credit card credentials: $\$ 35-135$, a set of online service account credentials: US\$19; a list of mobile phone numbers: $\$ 290-\$ 1,236$, and a list of landline phone numbers: $\$ 317-1,931$ [17]. Same consumer records may be sold unlimited number of times by copying. The unlimited number of sales and a large number consumers records can generate very large rewards. For example, the tmobile breach lost 45,000,000 consumer records. Each record comprises personal name, address, potentially Social Security Number, and at least one credit card. The data in each record is sufficient to enable a person to process a payment transaction. If the consumer records sold at only at the lowest cost of just $\$ 10$ and only about $1 \%$ of the records are sought by potential buyers, it would generate about $\$ 4.5$ million. For the leaked $864,500,000$ records from Facebook from 2013 to 2019, the hankers could get $\$ 864,500$ assuming that the records are sold at $\$ 20$ per record one time with $0.1 \%$ of consumers' data.

The useful duration of consumer identity data depends on use purposes. Some of the data are used for marketing and others are used in committing identity fraud. Most personal data such as phone numbers and addresses do not change at high frequencies, they may be sold five, ten, even twenty times. Other data such as Society Numbers, Birth day, personal working histories, education details, most biometric data cannot be changed at all, they would be sold for the 
consumer life times or even sold after the consumer's death. It is very possible that data brokers may validate personal identity data by comparing currently acquired data with those that they collected ten or twenty years ago. Consumer medical records may be sold at the price of $\$ 200$ [20]. Medical records of people with chronic diseases will be pursued by hospitals, medical practitioners, and drug companies. If consumer data is used in committing fraud and identity theft, its usefulness is perpetual, personal data can generate value even after the consumer's death. After a person lives for a life, he must leave many vestiges of his past existence. Using the identity of diseased persons is very common because the deceased persons cannot defend against the fraud: naturally damages will fall on governments, societies, and insurance companies. If the hackers use the data to produce values, there are unlimited potential to generate values. Due to limited sales times, the hackers or those trade the data would get very large rewards. It is obvious that personal privacy data do not produce immediate value to hackers, but may become useful when various pieces of personal data are combined to achieve a sufficiently large scope of personal information. Not all consumers are worthwhile subjects for producing rewards. When some of them generate wealth, reputation or political standing later, they will become subjects of identity theft. Full damages may be realized in any time in each victim's life time.

The second incentive is profits from selling consumer data by vendors. The beneficiaries include retailers, technology companies, social media, search engines and potentially media. The profitability of collecting consumer data depends on free trade of consumer data and unlimited resales or unlimited reuse values. It is anticipated that some hacked consumer records may be laundered as legitimate consumer data in the data broker market. For example, a hacker may hold himself out as a data broker, and sells hacked data to other data brokers. In the chain of transactions, the origins and nature of consumer data are lost. I must say that the 45 million data records leaked from t-Mobile and 20 million exposed from Apple most probably become sources of consumer data in the data broker market. Most of consumer data may be sold, traded, or used for marketing perpetually. In the U.S., all parties involving consumer data business have unrestricted freedom for selling and using hacked consumer data. Big social media, search engines, retailers, internet companies, etc. have inherent preference to see big consumer data broker market. There must be an industrial inherent bias in favor of seeing consumer data being hacked. All companies need consumer data to generate new leads for future business. It is possible that the 
values that advertisers can generate from search engines and social media companies may be much lower because the data have been overused.

\section{Eliminate Data Hacking and Data Collective Incentives}

I will show why consumer data hacking cannot be stopped by merely improved security measures. The internet commerce is based on the population model and trusted business systems. By following the population model, all users are treated in the same way as far as security is concerned. By following the trusted business model, the vendors can be fully trusted. This implies that consumers' account information, personal data, address, credit cards are stored in the plain text so that any employee of the vendor can read, copy and use them. Even account passwords are shared with the server. In this model, the vendors not only have the right to charge fees for their services, but own or control consumer data in an indefinite time and can even sell consumer data for profits. Since the server must validate consumer's identity, the password must be stored on the server or must be derivable from other information stored on the server. If a person knows security measures, password location, and key stretching method, the person can easily crack the security system. In order to get a better chance of success, hackers may seek help from insiders by providing sufficient monetary rewards. In such internet system, a successful cracking of one account $(\mathrm{N})$ is same as successful crack of all accounts. Moreover, each consumer record may be sold many times ( $\mathrm{T}$ ). The enhanced rewards come from two multipliers $\mathrm{N}$ and $T$. If the vendor has only one account which would be sold at $\$ 40$, the potential reword is $\$ 40 * \mathrm{~N} * \mathrm{~T}$. If the consumer record can be sold for 20 times, the hacker can generate $\$ 800$ for the life. If the system hosts 10 million consumer records, a successful hacking could generate $\$ 8$ billion of rewards for the population's life assuming that all records are good. When hacking can generate so much rewards, they can secure insiders' assistance by offering sufficiently high rewards. It is clear the prevalent data breaches can be stopped only by reducing the number of consumer accounts on the server system, using different account access methods, and a ban on trade of consumer data without consumer's concept, and imposition of actual liability for data breaches.

Reducing consumer account number is not an acceptable option for any business. A real security measure must use different encryption method for different users. One security measure is encrypting consumer data using consumerprovided encryption key and store the encrypted data on the server, and whenever the vendor needs to use the data, the consumer provides the encryption key that is used to decrypt the encrypted data to produce usable data. The vendor

Data Security, (c) JWu-v100 
will use the usable data once. The encryption key cannot be derived from stored information on the server, and thus insiders could not offer help. Now, the potential reward will decrease from potential $\$ 8$ billion to $\$ 800$. The potential reward will not be enough to pay for electricity for cracking the key and the hacker will not have the kind of money to buy insiders' help. Hacking will become a trade of the past. In addition, a rule is adopted to use the minimum consumer data for business transactions. This view is also suggested by other scholars [19].

The new security model is based on cost and reward analysis. I will consider the cost for hacking one single account by using brute-forcing user-key encrypted data. The average cost of electricity in the US is $\$ 0.12$ per $\mathrm{kWh}$. For a single server, it would need about $3741 \mathrm{kWh}$. That would be about $\$ 450$ per year for one machine. If a machine can do $10^{14}$ decryption attempts per second, the machine could do about $365 * 24 * 3600 * 10^{14}=3.15 \times 10^{21}$ decrypption attempts per year. The hacker would need to do $2^{255}$ decryptions on average to search the half of the key space so that it would need $2^{255} /\left(3.15 \times 10^{21}\right) \approx 1.84 \times 10^{55}$ machines. The total cost would be $\$ 450 \times 1.84 \times 10^{55}=\$ 8 \times 10^{57}$. Brute-forcing a 256-bit key would cost about $10^{44}$ times of the Gross World Product (GWP) or $\$ 63 \times 10^{12}$ [21]. This cost does not include the costs of depression of $1.84 \times 10^{55}$ machines in one year and labor costs. Similarly, cracking a symmetric 256-bit key used by AES by brute force would require a search of $2^{256}$ key space. The effort would require $3.67 \times 10^{55}$ years [22], but the average success time would be $1.83 \times 10^{55}$. In both computations, estimated costs do not include additional expenses that might arise from operational errors, machine failure, comparison time, and costs of human labors. The actual costs and time would be much more. All predictions strongly imply that brute-force attacks against 256-bit keys is unfeasible [22]. I also assume that brute-forcing even weak encryption method would be practical y unfeasible.

Even if the encryption is using a 64-bit encryption algorithm, the total time to search all keys by using a standalone server would be $t=2^{64}$ / $\left(3600 * 10^{14}\right) \approx 51$ hours. Based on experience, a one-trip internet interaction would take 1 second, depending on internet speed, server speed, server load or total connections, and the size of rendering page. By brute forcing over an internet connection, searching a 32-bit key or a 4-character key would take a total time: $\mathrm{t}=2^{32} / 3600 \approx 1.2$ million hours or 136 years. Those computations imply that bruteforcing by normal internet connections is unfeasible even for a 32-bit encryption algorithm or an encryption key like "h7el". There are all kinds of other huddles such as connection time limits, attempt limits, AL detection, etc. It is unfeasible to directly brute force encryption keys over the internet. Those computations im- 
ply that one workable strategy for hacking mass consumer database is finding the password database, getting the passwords or all information that could be used to re-build consumer passwords. Other possibility is exploring software design features and planting malware to bypass or disable server security features so that the hacker can get account data or passwords. Afterwords, the hacker can have years to access and acquire account data, most probably by automatic algorithms. If a system can use at least 128-bit encryption, encryption of consumer data by using a consumer's key will have a similar protection of "do-nottell your secret", absolutely the highest security measure.

\section{NEW TECHNOLOGIES FOR ALTERING DATA ECOSYSTEM}

\section{A. Use Minimum Data In Conducting Business Transactions}

I will explore what kind of consumer data are required for e-commerce. A start point is examining cash transactions. In a cash transaction, only things that must be trusted by two sides are currency authenticity and product amount and quality. Such transactions do not require anything such as personal address, identity, phone number, email address, and street addresses. There is no need to collect any of those items if cash can be authenticated and products can be inspected. This model does not work well in two kinds of situations. In one kind of situation, the products in exchange are very complex and thus cannot be determined by an on-site inspection. Thus, what is required is more time for the buyer. In another kind of transactions, the seller will sell products or services on monthly basis and the seller needs to secure that timely payment will be made in given time intervals, like an utility contract between a consumer and a utility company. In this kind of arrangements, the seller must ensure that buyer will make timely payment each time. To enforce each payment, the seller needs to know the person and thus there is a need to collect information on the personal address, phone number, birthday, and payment mode. Those information is merely incidental to the need to enforce the agreement or secure the last payment in the event that the agreement ends. Most studies on trust are revolved around vendors [23-25], some measures such as third party assurance [24] and legal remedies [25] would be similarly applied to consumers. Indeed, the most usable measures are evaluating consumer's credit records and demanding a reasonable deposit for the consumers with poor credit ratings. Most personal information can be avoided if a proper arrangement can be made. This minimum

Data Security, (c) JWu-v100 
data principle for building a healthy data ecosystem implies that a total ban of the broker market could kill economy because businesses do need the minimum consumer data to complete each business transaction and consumers must be publicly visible like real humans in streets. A future data regulation for Congress is to find the optimum point between free access to consumers and eliminating data that would be used by identity thieves and aggressive product promoters. I will explore how an installment payment can be arranged without collecting unnecessary personal information below.

\section{B. New Technologies Based on Oldest and Timeless Idea}

The new technologies are based on the oldest principle: that the safest measure is not sharing one's private information with anyone. The technologies to embody this principle can be described as follows:

1. Upload personal data in an encrypted form to a server and store the data in an encrypted form on the server. This is a one-time effort.

2. Send an authorization to conduct the business transaction such as a making a payment. It normally takes a few seconds to complete.

3. Server responds with an acknowledgment.

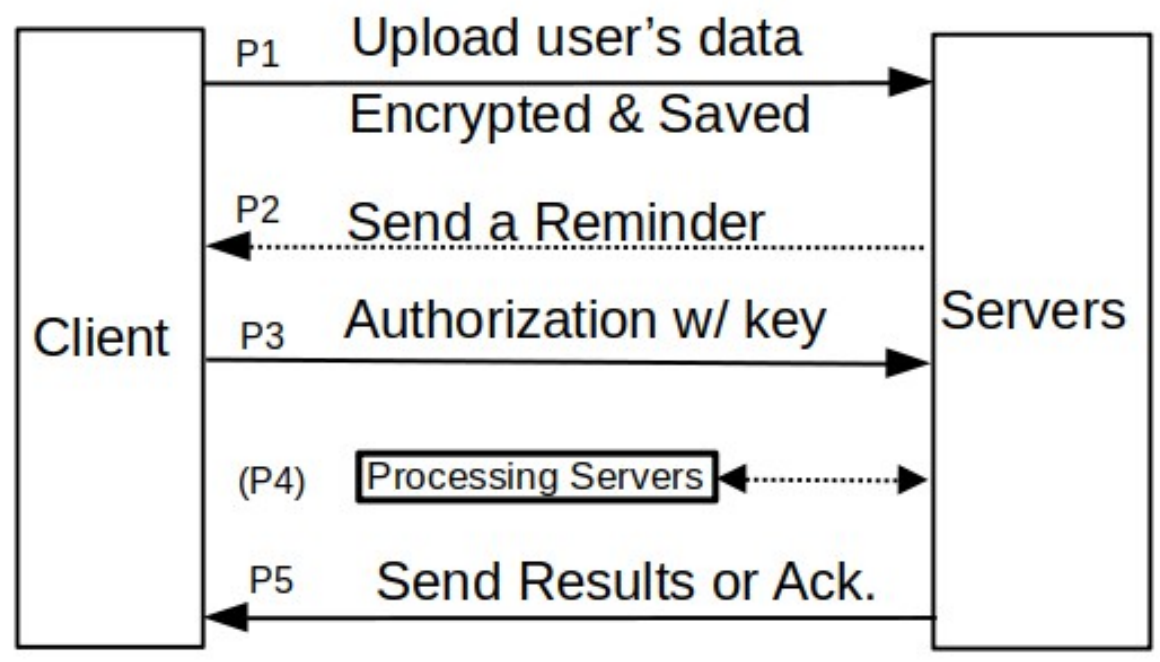

FIG. 1. The Process For Processing A Payment Using Encrypted Consumer Data. 
For example, a payment can be completed by using the following user interfaces (FIGS. 2-4):

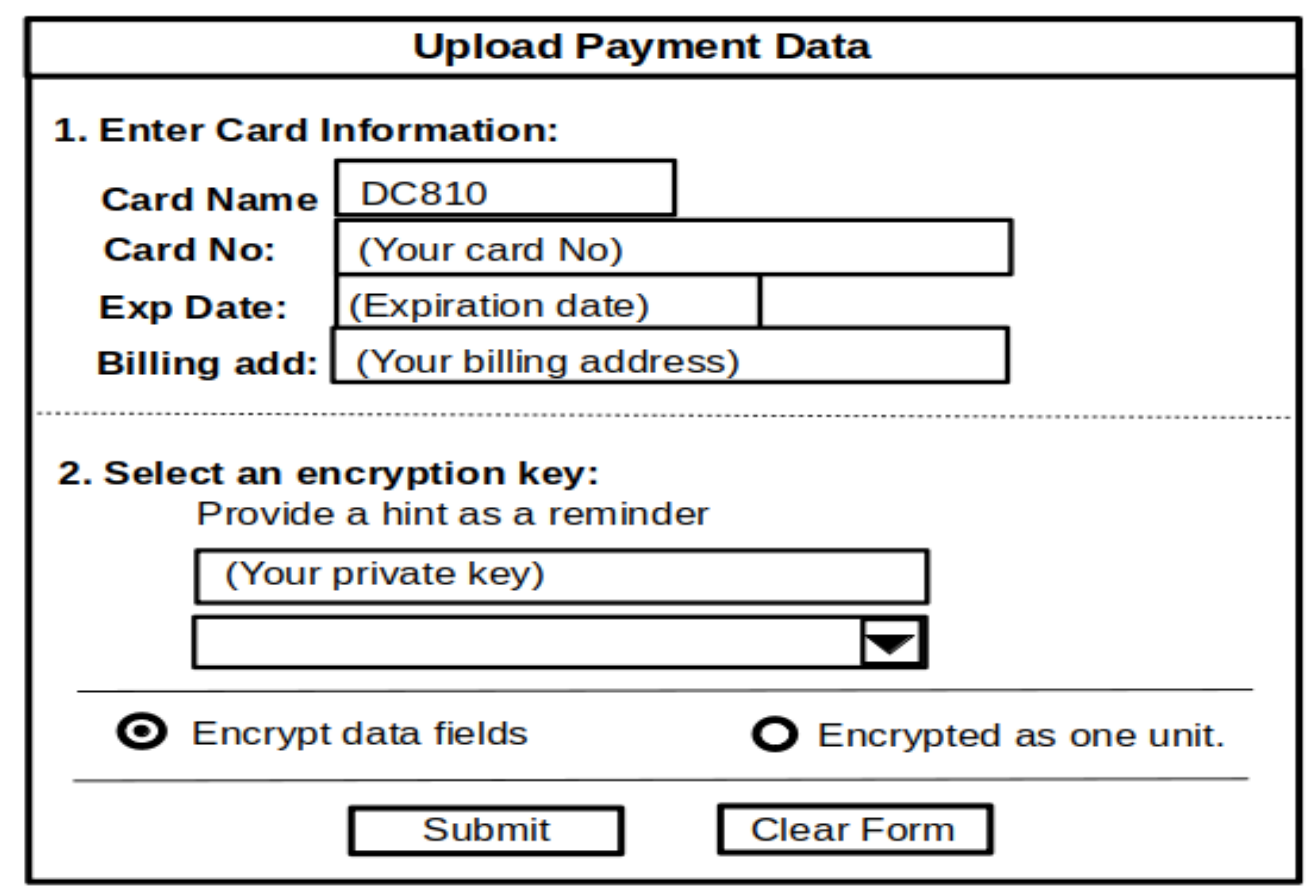

FIG. 2. A Exemplar User Interface for Uploading Payment Data.

All sensitive information as shown in FIG. 2 is encrypted on a client computer or a server by using a user-provided encryption key and stored in encrypted form on the server. The consumer data are not usable by the server without the user-provided key. Now, if the consumer wants to make a payment which may be initiated by the server.

In making the payment as shown in FIG. 3 , the consumer or the user is required to provide only an encryption key or the location of the key, and the amount (if the user wants to override the amount in the setup). The server gets the encryption key and uses it to decrypt the stored payment data to produce usable data and process the payment on this server or a connected server. The server then provides an acknowledge message as shown in FIG. 4. 


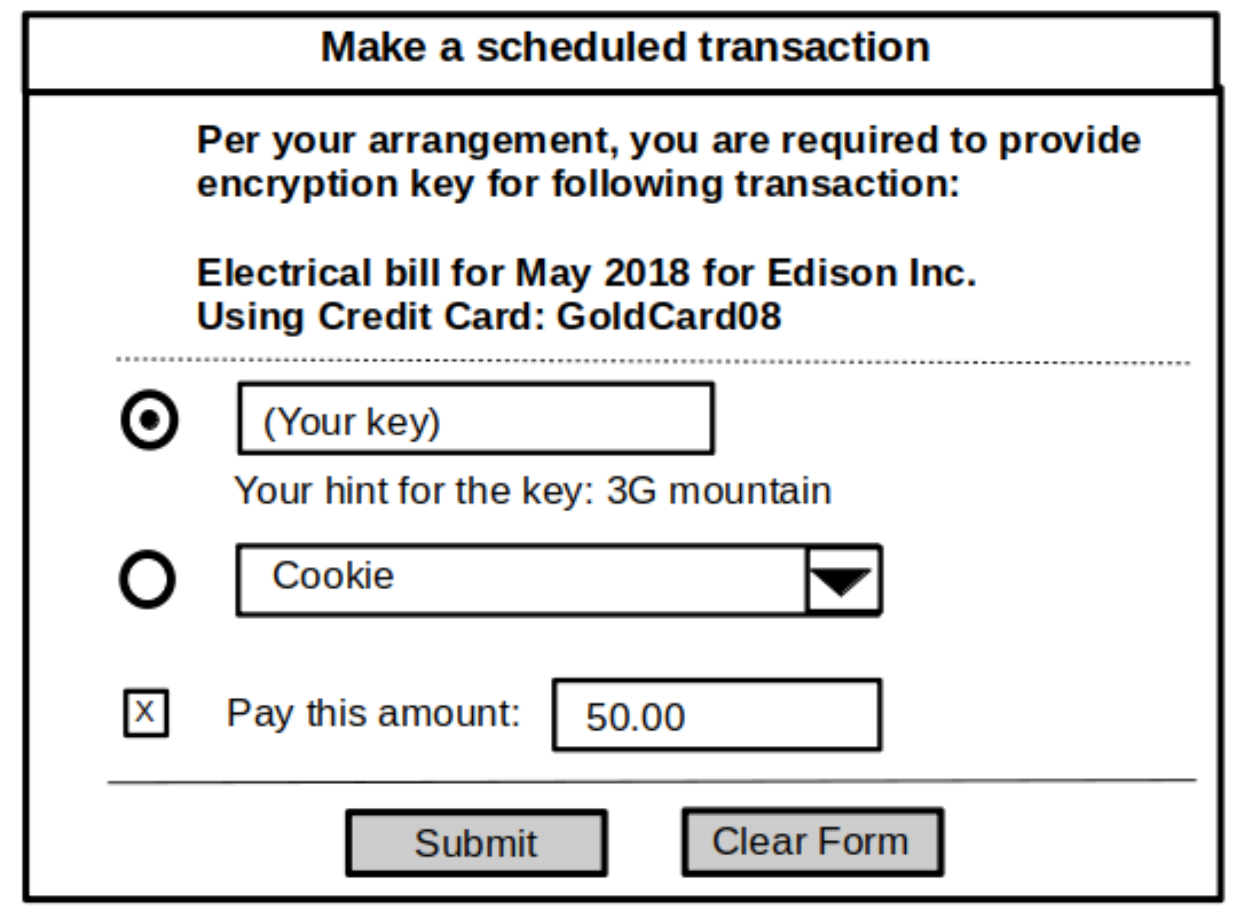

FIG. 3. A User Interface for Authorizing a Payment.

You have made the following transaction successfully:

Type transaction: $\quad$ Electrical bill for May 2018

Payee:

Amount:

Edison, Inc

$\$ 50.00$

Date: June 28, 2018

Confirmation No: $\quad$ XYZ125000

Next Payment: $\quad$ Request arrive on XX

Close

Print

FIG. 4. An Exemplar User Interface Showing An Acknowledge of Payment.

Data Security, @ JWu-v100 
All sensitive data on the server are encrypted by using different keys and potentially using different encryption algorithms. Hackers will not brute-force consumer data from an account for about $\$ 50$ to $\$ 200$ potential return per success or $\$ 800$ for the person's lifetime. To hackers, all data from the server's database is useless. This concept will lead to a new data security ecosystem: insiders-assisting hacking will be out of business.

\section{Feasibility of The Technologies}

In conducting a business transaction, personal data, payment data, and most confidential data are not the type that can lose permanently. If the encryption key is lost, the maximum burden is re-creating the transaction data. The encryption key does not require very high strength. For hackers and thieves, a key like a street name, an object name, animal name, or any combination is enough to defeat the incentive of cracking the encryption key because the hacker is not in a position to know anything about the consumer and other consumers. Such a key can be effective even if the keys are grossly insufficient for other security purposes. Due to impossible to brute-force an encryption key in practice, the technologies can achieve the benefits of the "do-not-tell" principle that will never become obsolete. The new technologies can put an end to the prevalent consumer data breaches, which were created, promoted and proliferated by the flawed population model. The technologies have following advantages:

1. Completely eliminate the incentives for hackers to crack an online server system's security measures to get millions of account data for unlimited sales. On the server, each consumer data record is encrypted by a user-provided key. A hacker cannot hack the system to crack millions of consumer data records.

2. A payment or other transaction can be conducted in seconds, even faster than using two-factor authentication. The time can be even shorter than what is needed to make a regular payment. For other transactions, the time is just as short as typing or pointing a location of the encryption key.

3. Increase consumers' confidence in using debit cards, banking cards, visa cards, master card, American express cards, etc. in all kinds of commercial environments. When consumers are finally assured that their personal data and financial information cannot be hacked, they will be more liberal in entering transactions.

4. Reduce the burden on the businesses to secure customers' data online; thus, small and middle-sized businesses can accept and use credit cards, banking cards, personal information, etc. without exposing their liability. As long as the 
consumer data are encrypted by 128-bit or higher, the business owners do not need to worry about data security. The responsibility to control consumer information is given back to consumer. There is no need to get this hot potato.

5. Reduce consumer's burden in learning new security technologies, changing account passwords, and updating software. Each new technologies will improve security for a short time. When hackers figure out how to crack the new technologies, the system has to seek further new technologies. Each new technology will require the consumer to learn security methods. At times, they will get into trouble due to their own mistakes. Nearly all consumers have been tired of the never-ending security upgrades that can never end the nightmare of huge data breaches.

6. Reduce the access interruptions caused by consumers' mistakes, hardware incompatibility and software incompatibility, etc. which can be caused by or traceable to newly introduced security measures. After consumers have used the same systems for long times, they have learned how to address certain interruptions. When they run into a new problem with an upgraded security measures, they may have no time to address the problems and can be negatively affected due to all special circumstances.

7. Reduce downtime that can be traced to complex and never-working security technologies and help employees to reduce the burden of navigating multiple layers of security measures that never work. In some working environments, employees may be required to pass three to four layers of login, hardware authentication, etc. None of them can keep out real hackers. Most security measures just kill productivity and cause their clients to spend more money.

8. Provide the best protection for "consumer with lost identity." After a huge number of consumer data breaches, most U.S. residents have become public persons, whose social security number, birthday, address, and other personal data have been stolen in one or more data breaches and those identity data are available for sales in the black market. Most of them have not been hit by fraud or criminal activities because they are not in the circumstance of generating values yet. One big problem is that they cannot have their personal identity protected anymore. By using the new technologies, the consumer can create a new personal identify information. Also, when authorization can be made only by using a user-provided key, hackers with his personal information cannot bypass. For all those who have lost identity data, the future authentication must be based on their secret keys but not their birth days and social security numbers.

Data Security, (c) JWu-v100 
9. Prevent more pipe-line disasters from misuse of personal biometric data such as DNA, blood type, fingerprints, face looks, etc. None of those insider-assisted data breaches can ever be stopped by using personal biometric data. Since biometric data must be stored on a server which can be accessed from the internet, they can be stolen. After biometric data of consumers on an internet system are stolen, they will be copied, passed from person to person, and are available for sales in the black market. Under current technologies, victims cannot protect their identities any more because they cannot change their biometric data. Any claim that biometric data can improve data security is a wish that must be wrong. There is no reason such measures can succeed. The new technologies will give the lost-identity persons a second chance to protect their identities and financial data in a different way.

The technologies help environment protection and promote sustainable economy. It will stop massive wasteful human activity cycles that start with leaking consumer data, fixing all kinds of problems attributable to damaged personal credits, lost money and properties, damaged reputation and damaged personal health, restoring personal credits, recovering financial loss, restoring personal health, catching and prosecuting thieves, and jailing thieves. The massive activities that resolve around leaked consumer data records do not produce any net benefits to victims and societies. All of those activities damage victims, society, governments, and planet.

The use of the technologies alters the internet convention that the business has a total control over consumer data. To make this system workable, the rights and obligations on managing consumer data must be modified by agreements, common understandings, regulations, trade regulations, statutes, state laws, local laws, etc. The merchant may use consumer data only when there is a need. It does not own consumer data. After use of the user's data, the usable data is deleted or discarded. The data in the usable form may be saved on the server only for a limited time or just sufficiently long for completing an intended business transaction; and the consumer is obligated to provide an encryption key to decrypt stored data for each transaction per their agreements. In a payment system, the merchant must use a gateway, a payment processor, a central computer connected to a bankcard network or automatic clearing house, etc. Each of involved parties follows the same rule.

\section{Impacts of Possible Consumer's Errors}

A common online fraud is caused by consumer's errors in response to an fake email. For example, a consumer might receive an email from a bank that 
urge the consumer to approve a pending transaction. If he did not ascertain the authenticity of the email, he might try to log into the account. In this process, the consumer provides his log-in name and password end up with a message to log back next day. If the consumer did not know that he was responding to a fake website for harvesting his log-in data, his credentials information will be used to access this banking account shortly. By using the new security mode, the most regular transaction is sending an authorization for a scheduled business transaction such as making a payment. This information contains only an encryption key for data storage purposes. If the consumer has responded to a fake payment reminder, the consumer will compromise only the encryption key. Even if the key is acquired by a hacker, the consumer just loses the benefits of data encryption in storage. The key itself has no value like other personal data. It can be changed easily in a few minutes. Moreover, even if a good number of people have such compromised keys, the net effects are like a few percents of accounts data are stored in plain text. The normal password protection against unlawful access and data transmission protection are still in force. It cannot change the data ecosystem: cracking his account is still unfeasible or a money-losing effort.

Another issue is what is the impact of forgetting an encryption key on consumer and businesses. First, consumer data are not the kind of data that can be lost forever. They are not presented to a vendor for permanent storage in nearly all transaction environments. If a consumer has lost his key, he could re-submit his personal information for encryption. The scope of impacts is only on the sensitive information that the user wants to protect. Thus, the burden is very limited. Because the encryption key is not a real private data, and has no value in the data broker market, the key can be stored on a personal computer, a cellular phone, and/or recorded in personal books. It's main purpose is creating a data ecosystem that make hacking a money-losing trade. Since most hackers are remote from the consumers in most situations, it is impossible for the hacker to know about anything about the consumer. Even if a consumer selects an encryption key based on his birth day (even though a bad idea), home street name, school name, car model, etc, it is not what the hacker knows. The hacker faces the reality he must brute force each consumer record with no useful hints at all. An expected maximum reward in the amount of $\$ 800$ is a lost deal.

\section{E. Application Limitations of the Technologies}

The technologies cannot be used to protect data originated from the server. The technologies cannot be used to protect bank transaction data that are generated from the banks. Similarly, the technologies cannot be used to protect 
vendors' sale records such as items, charges, sale dates, etc. Fortunately, those data are not the kind of data that hackers would use to generate money. The utility of the technologies is based on the assumption that the server is controlled by the business vendor so that tempering of server software is not allowed. If the whole server software can be tempered, nothing can ever protect consumer data.

The technologies may be used to protect consumer data that are used by business. The technologies can be used to protect consumer data for utilities companies such as gas companies, electricity companies, water companies, internet companies, retailers, wholesales stories, chain stories, banks and financial institutes, drug stories, credit report agencies, hospitals and health care providers, insurance companies, technical companies, large restaurants, chain restaurants, airlines, bus companies, transits, metro, schools and universities, government agencies, and any other vendors that have needs to keep credit cards, banking cards, and personal data for convenience of the companies. If the technologies are used, banks reduce financial loss from credit card frauds, vendors avoid liabilities for leaking personal and financial information, consumers save time on aftermath effort to restore personal privacy and changing passwords, consumers do not need to keep learning new security technologies that cannot work for a long time, and employers can see improved work productivity due to reduced interruptions by failed security measures. The technologies may be used to protect staff's personal data. Official data for sensitive federal agencies may be protected if security responsibilities can be allocated to specific persons.

When the technologies are deployed, consumers should be reminded that the vendor cannot recover lost encryption key. If a user wants to keep a document or information as the sole source of storage, the user must keep the encryption key permanently. Due to separation between the consumer and the hacker in space, the encryption keys do no need to have high security strength. If data must be protected permanently, the consumer should keep the key in at least two other sources. To avoid that the key is sold by employees of key keepers, the use purpose of the key should not be disclosed to anyone.

\section{NEW TECHNOLOGIES CAN PREVENT ALL DAMAGES}

\section{A. Existing Technologies Cannot Stop Mass Data Breaches}

Data Security, (c) JWu-v100 
All existing technologies are based on trusted systems, thus they must have three vulnerabilities: similar or unique exposures of data risk, insider attaches and insider collusion, and discovery by abusive legal process. The real reward is about $\mathrm{N}^{*} \mathrm{~T} *$ Selling price. The two multipliers ( $\mathrm{N}$ and $\mathrm{T}$ ) and the secured trust system designs are the main reasons for failure of all existing security measures. All existing technologies could not protect data storage security. All authentication protocols such as multiple-factor validation [27] and email or phone code and new technologies are ineffective against insider-assisting hacking and in abusive subpoenas. For the same reason, security tokens cannot protect against insider acts and abusive legal subpoena. When thieves cannot get access to secure properties, there is a chance that the thieves will stalk and assault the property owner to gain access. If the item is secured with a biometric device, the damage to the owner could cost more than the secured property. For example, in 2005, Malaysian car thieves cut off a man's finger when attempting to steal his Mercedes-Benz S-Class [30]. If biometric data are stored on a server, hacking and acquiring the biometric database from the server would enable thieves to create artificial fingers, faces, etc to gain access in target attacks. As long as the server has copies of consumer's data to be used for comparison, a hacker must be able to get the data in target attacks. The most recent technologies are using AI in Cybersecurity [31]. AL may help organizations to detect, predict and respond to cyberthreats in real time using machine and deep learning. While they may mitigate access risks, its capability would depend on design of the tools. For those know how those tools work, they can avoid their detection. I predict that they might cause unintended problems and unwanted interruptions. The biggest future risks are acquisition of personal biometric data. Biometric data can be used only if biometric data can be secured by $100 \%$. Steeling of biometric data will turn such a person into a public figure who has lost personal secrets.

\section{B. Distinctive Damages to Corporations, Societies and Consumers}

\section{Damages to Corporations Responsible for Data Breaches}

Mass data breaches can cause overwhelming damages to business, government, institutions and consumers. I will show that the magnitude of damages, the duration of damages, and the scope of damages are grossly underestimated in the literature.

Most studies on damages caused by mass data breaches focused on only the business side. When a system is found compromised, the owner must take measures to stop it. This can be a complicated process. An IBM study found that 
the average total cost for a data breach is $\$ 3.86$ million [32]. While the average cost per lost or stolen record was $\$ 146$ across all data breaches, those containing customer PII cost businesses $\$ 150$ per compromised record [32]. The damage to corporation may come from a combination of reduced customer traffic, costs associated with responding to the breach, and the need to establish reserves against future legal judgments. In Target data breach, the damages reduced Target's earnings before interest and taxes by nearly 30 percent - a reduction of the company's earnings by $\$ 1.58$ billion, from $\$ 5.52$ billion for the year before the attack to $\$ 3.94$ billion for the year after it. Costs directly related to the attack, including settlements of lawsuits, totaled \$292 million [33].

The biggest damage is slowly realized by identity theft which must be fueled by data breaches. This kind of damages cannot be determined because leaked data can be laundered to gain new identity on the data broker market. Even in 2000, FBI estimates that identity theft affects 350,000 to 500,000 victims in the U.S. annually [34]. More than 406,000 people reported that someone submitted a fraudulent government document under their name [35]. From 2019 to 2020 , the U.S. experienced a $311 \%$ increase in victims, with a total payout of $\$ 350$ million [36]. This huge increase has been attributed to the practices of working at home. It seems to suggest that control of employees' behavior is the most important factor. In most working environments, employees must access client data or consumers data. What is changed is the degree of control of business over their employees. In this working environments, the business data must move through more internet wires and more computers, there are more likelihoods the consumer data are viewed by people at homes and there is possibility that a small number of employees harvest consumer data. The real danger is that when an employee can access to the whole customers database, nothing can stop the employee from getting customers' records. However, real and biggest damages have not been studied. There must be long lags between data breaches and strikes of identity theft caused by leaked data on consumers.

\section{Damages To Societies and Governments}

Identity thieves scavenge through garbage, steal and redirect mail, use database internal access, and surf the Internet for personal information [34]. The travel paths of consumer data originated from data breaches cannot be determined by any method. Some of hacked consumers' data may be sold in websites. A consumer's records may be exposed by different mass data breaches. Personal data such as address, phone number, email address may be exposed by data breaches of social media companies like Facebook; sensitive personal finan- 
cial data may be exposed by data breaches of banks, credit card services, and credit card companies; driver license numbers, social security number, and birthday, marriage status may be exposed by data breaches of government agencies. It should be assumed that most of the consumer data would be channeled to the underground data markets or data broker market. While hacking may be motivated by various reasons, the primary motivation is obtaining financial rewards.

The crimes involving use of consumer data include exploiting weakness in specific technologies and information systems, financial scams, other crimes, facilitating crimes, avoiding arrest, repeat victimization. Victims may be children, the elderly, the diseased, companies, institutions, governments, the justice system [34]. There are all kinds of unforeseeable impacts by crimes involving identity theft. Even general public safety can be threatened by identity theft when offenders pose as "qualified" or trained professionals such as doctors and individuals with Commercial Driver's Licenses [34]. There are many other possibilities: some people may be imposed as lawyers, police officers, judges, government officials, etc. Damages from such activities can never be included in the all kinds of damage studies.

I will cite some of the costs to societies, government and consumers. Between 1995 and 1997, the Secret Service estimated that actual costs associated with identity fraud arrests were $\$ 442$ million, $\$ 450$ million and $\$ 745$ million, respectively; Its best estimate of the average cost for a financial crimes investigation in 2001 was $\$ 15,000$, but such cases vary; FBI estimated the average cost of an investigation by its white-collar program to be $\$ 20,000$ between 1998 and 2000; The people may use fake identy to file questionable tax returns; The Social Security Administration/OIG reports that the number of social security misuse investigation increased from 305 in 1996 to 1,153 in 1997. With regard to the costs of corrections, the Bureau of Prisons reported that the cost of operating a minimum-security facility, where most white-collar offenders reside, averaged $\$ 17,400$ per inmate in 2000. Offenders are then supervised in the community by federal probation officers for a period of 3-5 years at an average cost of $\$ 2,900$ per offender - a cost that does not include any special conditions such as community service, electronic monitoring or substance abuse treatment. Since identity theft are involved in various crimes, identity theft must be responsible for a portion of those institutional costs. The costs to governments may be very large because the identity theft are prevalent. I believe that many known identity theft may be an essential element of committed crime even though the roles of consumer data are unknown or involved identity theft is not reported. 


\section{Damages to Consumers}

Some individual victims do incur financial costs that businesses do not cover. One study found that the average out-of-pocket expenses reported by victims were between $\$ 30$ and $\$ 2,000$, but this estimate does not include any lawyer's fees that were incurred. The average loss to victims in this study was $\$ 808$ dollars, but most estimated spending around \$100 [38]. The average amount of out-of-pocket expenses for all types of victims, as reported by the FTC, was \$500; however, the average out-of-pocket expenses for victims in cases where a new account had been opened were $\$ 1,200$ [37]. Overall, one estimate provided by the National Fraud Center, "conservatively" estimates the costs of identity theft to individuals to be $\$ 50$ billion dollars per year in 2000 [39]. This amount must be much higher today. Losses caused by application fraud are forecast to increase in 2021 to $\$ 721.3$ billion [40]. This study narrowed the identity theft definition to include only application fraud, where criminals used a victim's identity to open a new account of some type, and account takeover, where an account is taken so criminals can steal money or access rewards. Examples of accounts include rewards accounts for airlines, hotels, or merchants; insurance policies; and other accounts. The total number of people reported identity theft is $4,772,748$ for 2020 . This figure does not include unreported victims, dormant victims the impacts on who have not been perceived, and future victims whose financial loss may appear years later. A survey conducted by Aite group in 2020 found that in the past two years, almost half (47\%) of U.S. consumers surveyed experienced identity theft; well over one-third (37\%) experienced application fraud (i.e., the unauthorized use of one's identity to apply for an account), and over one-third (38\%) of consumers experienced account takeover (i.e., unauthorized access to a consumer's existing account) [40-41]. I have no reason to question the approximate validity of the survey by considering the magnitude of consumers records that have been hacked in the past.

One biggest problem is cost time (in the form of hours spent on the phone trying to resolve it) and money (in the event that the criminals have gained access to one or more of consumer accounts). The statistics from The Aftermath study show: $26 \%$ of respondents had to borrow money from family or friends; $22 \%$ took time off work; $15.3 \%$ sold possessions to pay for expenses caused by their identity theft, and $6.7 \%$ obtained a payday loan. Much severe damage is emotional distress. It is an annoyance to spend so many hours dealing with the problems; $66 \%$ of respondents experienced fear regarding their personal financial security; $53 \%$ felt a sense of powerlessness or helplessness; and 7\% reported feeling suicidal. $75 \%$ of identity theft victims reported that they were severely 
distressed by their experience. One-fourth of respondents sought professional help to manage their identity theft experience-either going to a doctor for their physical symptoms, or seeking counseling (therapy, group therapy, or some kind of support in that manner for the emotional implications). Some people may suffer severer damages due to their unique circumstances. They may be denied in job application, rent application, business opportunities or whatever they are seeking. When it gets to the level of things like a romance scam, it is embarrassing to talk. In all leading studies on mass data breaches, the largest damages to consumers escaped from scrutiny obviously because hacked consumer data can easily be laundered. Based on my reading of civil litigation in the claim arising intentional infliction of emotional distress, the damages to consumers is not about $\$ 40$ average price or high bound of two hundreds, it can easily hit millions for a good portion of victims who are more vulnerable due to their unique circumstances. The massive damages to emotion and personal dignity are not counted as the total loss of $\$ 721.3$ billion of identity theft.

Most of the recoverable damages will fall on insurance companies, federal government, state governments, consumers, and non-internet companies (by way of productivity loss and interactions of jobs, etc) in very long time. It is not overstate to say that identity theft in the U.S. is becoming a national emergency that must be addressed from multiple angles. Because consumer data have perpetual life, consumers who have previously been affected by identity theft are at a greater risk for future identity theft and fraud. Among surveyed consumers, three out of every ten people confirmed it [41]. It is expected that when identity thieves have collected more complete information about particular consumers, the thieves are more likely to able to extract values from the identity data of the consumers.

\section{Existing Technologies Cannot Prevent Mass Data Breaches}

The damages to corporations, societies and consumers are different in kinds and amounts. In the current legal environment, corporations leaking consumer information do not need to pay full damages to consumers because the legal system fails to see that real damages may take years to decades to realize. Consumer data can persist even after the death of the person. While consumer data can be sold perpetually, the adverse impacts of leaked data are determined shortly after the data breach. It is impossible to prove that consumer data can find their way into data broker market. No body can prove to what extent the mass data breaches by Microsoft, Google, Facebook, Apple, T-Mobile, etc. have promoted identity theft. High-profile data breaches continue to threaten business 
with most losses landed on consumers and societies. In 2021, more than 280 million Microsoft customer records were left unprotected on the web in January. By March, the U.S. Cybersecurity and Infrastructure Security Agency advised all organizations across all sectors to address Microsoft's email server vulnerabilities [40]. If hackers are able to find the security vulnerability, it is possible that any of the Microsoft servers may be the subject of later attacks.

The consumer data breach nightmare can be traced to the business model and the practices of big tech companies. Healthy technologies landscape would depend on the timely technical change-over; but after big tech companies have developed appropriate security technologies, they will never give up obsolete and useless technologies but do everything to prevent start-ups from replacing technologies [42]. It is indisputable that most mass data breaches can be traced to technical companies or fortune companies in 2020 to 2022 such as Microsoft, Google, Facebook, Apple, etc. They can avoid paying full damages to consumers and societies and pay nothing to governments and other non-internet companies. They can continue to inflict more damages to societies and consumers because trade articles fail to explore the magnitude of damages of breached data in the entire time. For example, Facebook obtains its operational revenue by generating a massive number of identity-lost consumers and then shift all damages to governments, societies and consumers without paying meaningful amounts. While the data in hacker's hands will be on sale indefinitely, Facebook does not need to pay for all future and delayed damages. It may pay a nominal dmages, and then continue collecting personal data to earn advertisement revenues and continue exposing consumers data again and again. All of its gain is achieved by inflicting damages to governments, consumers, insurance companies and non-internet companies. Big tech companies should have long ago realized that no technologies can ever stop insider-assisted hacking due to the large rewards ( $\mathrm{I}=\mathrm{N} * \mathrm{~T} *$ Price). The payments in those settlements of data breaches are fractions of expected full damages. The never-ending data breaches is the reason for seeing a state of national identity theft emergence: when almost half of the surveyed people have lost identities, I can safely assume that most U.S. residents have lost their identities. It is only a matter of time and opportunities for most of them to feel delayed identity theft strikes. Even if new technologies were used immediately now, the consumer data broker market is banned, and collection of consumer data by cookies are strictly regulated, consumer identity theft will continue rising, damaging consumers, governments and societies in the scale no one has predicted before. 
Despite the repeated failure of data security measures, consumers still use the services of the big tech companies. One obvious reason is that consumers have been told that no technologies can really stop data breaches. Consumers have been told that security can be improved only incrementally. Consumers' trust in the big tech companies is based on competence, openness, concern, and reliability [43]. In the past, when no viable security measure exists, consumers cannot challenge any of those elements. After I have found the root cause of data breaches and the negative role of large tech companies, consumers' trust in the companies will collapse. Failure to address the data security will discredit their openness. Failure to stop future data breaches will be perceived as incompetency and poor reliability of their products and services. In a competitive market, gaining and holding a loyal customer base is a key corporate challenge, and how the company backs up its commitments to products and services largely determine whether the customer will remain loyal or switch to another company [44]. The tech companies cannot expect consumers to continue supporting their business while keeping inflicting damages and escaping from most liabilities. The cost for addressing data breaches is the highest in the U.S. because loss of trust does translate into a much higher cost [45]. Diminished trust of customers, business partners, and regulators all will work against the companies.

\section{Conclusion}

The frequent mass data breaches can be traced to the influence of the trusted business system and population-based approach. In such a system, successful cracking of one account is naturally rewarded by expected sale price multiplied by account number and unlimited sale times. The potential rewards to hackers for each successful cracking of a business server are so larger that no technologies can ever stop insider-assisting hacking. The data security industry has not pay attention to the perpetual impacts of leaked consumer data and the delayed damages to consumers, governments and societies. The existing technologies cannot solve the problem while more and more U.S. residents have lost their identities. Based on the above analysis, it is fair to conclude that social media like Facebook achieves its revenues by inflicting overwhelming damages to consumers, governments, institution of justice, insurance companies, and most non-internet business corporations. The tech corporations pay only fractions of real financial loss to consumers also because they refuse to acknowledge damages to undiscovered victims and future victims. Identity theft is becoming a national crisis. A workable solution to this problem must be drastic. The new technologies, which comprise encrypting and storing encrypted data and using

consumer data upon user-provided key, can change data ecosystem and thus 
eliminate hacking incentive and abuse of consumer data. The technologies can give a second chance to the majority of U.S. residents who have lost their identities.

\section{REFERENCES}

1. Ellis J. Distributed Access To Valuable And Sensitive Documents And Data. US Patent Application: 20070220614

2. Pennington R, Wilcox D. The Role of System Trust in Business to Consumer Transactions. Journal of MIS. 2004;20(3):197-226.

3. Das TK, Tetig B. Between trust and control: Developing confidence in partner cooperation in alliances. Academy of Management Review. 1998;23(3): 491-512.

4. Hart PJ, Saunders CS. Emerging electronic partnerships: Antecedents and dimensions of EDI use from the supplier's perspective. Journal of Management Information Systems. Spring 1998;74(4):87-111.

5. Hawes JM, Mast KE, Swan JE. Trust earning perceptions of sellers and buyers. Journal of Personal Selling and Sates Management. Spring 1989;9(1):1-8.

6. Gefen D. Reflections on the dimensions of trust and trustworthiness among online consumers. DATABASF for Advances in Information Systems. 2002;33(3):38-16. Doi: 10.1145/569905.569910

7. Doney PM, Cannon JP. An examination of the nature of trust in buyerseller relationships. Journal of Marketing. 1997;61(2):35-51.

8. Ba S, Pavlou PA. Evidence of the effect of trust building technology in electronic markets: Price premiums and buyer behavior. MIS Quarterly, 2002;26(3):243-268.

9. Chiravuri A, Nazareth D. Consumer trust in electronic commerce: An alternative framework using technology acceptance. In D. Strong and D. Straub (eds.), Proceedings of the Seventh Americas Conference on Information Systems. Atlanta: AIS. 2001, pp. 781-783.

10. Hoffman DL, Novak TP, Peralta M. Building consumer trust online. Communications of the ACM. 1999;42(4):80-85. 
11. Jarvenpaa SL, Tractinsky N, Vitale M. Consumer trust in an Internet store. Information Technology and Management, I. 2000;1(2):45-7.

12. Lee MKO, Turban E. A trust model for consumer internet shopping. International Journal of Electronic Commerce. Fall 2001;6(1):75-91.

13. Wikipedia. List of data breaches. Accessed from https://en.wikipedia.org/wiki/List_of_data_breaches

14. Wikipedia. Data breaches. Accessed from https://en.wikipedia.org/ wiki/List_of_data_breaches

15. Privacy Rights Clearinghouse. Data Breaches (database). Accessed from https://privacyrights.org/data-breaches

16. Song V. (January 17, 2019) Mother of All Breaches Exposes 773 Million Emails, 21 Million Passwords. Gizmodo. Retrieved from https://gizmodo.com/ mother-of-all-breaches-exposes-773-million-emails-21-m-1831833456

17. Trendmicro. The Global Black Market Prices: These are some of your personal data and their corresponding prices: https://www.trendmicro.com/ vinfo/us/security/special-report/cybercriminal-underground-economy-series/ global-black-market-for-stolen-data/\#section-2

18. Tucker C, Neumann N. (May 01, 2020) Buying Consumer Data? Tread Carefully. Analytics And Data Science Harvard Business Review. Accessed from https://hbr.org/2020/05/buying-consumer-data-tread-carefully

19. Martin B. The Unregulated Underground Market for Your Data: Providing Adequate Protections for Consumer Privacy in the Modern Era. 105 Iowa L. Rev. 865 (2020).

20. Max E. How Companies Turn Your Data into Money, PCMAG (Oct. 10, 2018, 8:00 AM), https://www.pcmag.com/article/364152/how-companies-turnyour-data-into-money [https://perma.cc/Y8SN-TS53].

21. Stockexchange.com How much would it cost in U.S. dollars to bruteforce a 256-bit key in a year? https://crypto.stackexchange.com/questions/1145/ how-much-would-it-cost-in-u-s-dollars-to-brute-force-a-256-bit-key-in-a-year

22. Wikipedia. Brute-force attack. https://en.wikipedia.org/wiki/Bruteforce_attack\#Theoretical_limits 
23. Hart PJ, Saunders CS. Power and trust: Critical factors in the adoption and use of electronic data interchange. Organization Science, 1997:8(1):23-42.

24. Kimery KM, McCord J. Third-party assurances: mapping the road to trust in e-retailing. Journal of Information Technology Theory and Application, 4. 2002;4(2):63-82.

25. Sitkin SB, Roth NL. Explaining the limited effectiveness of legalistic "remedies" for trust/distrust. Organization Science, 1993;4(3):367-392. (legalistic remedies often have limited effectiveness in addressing trust problems in organizations)

26. Schurr PH, Ozanne JL. Influences on exchange processes: Buyers' preconceptions of a seller's trustworthiness and bargaining toughness. Journal of Consumer Research. 1985;11(4):939-953. Doi: 10.1086/209028 (a seller's expected trustworthiness-plus-toughness in bargaining led to high levels of buyerseller cooperation and agreement and a higher level of buyer concessions.)

27. Wikipedia. Multi-factor authentication. Accessed from https:// en.wikipedia.org/wiki/Multi-factor_authentication

28. Wikipedia. Security token. Accessed from https://en.wikipedia.org/ wiki/Security_token

29. Wikipedia. Biometrics assessed from https://en.wikipedia.org/wiki Biometrics\#Danger_to_owners_of_secured_items

30. Kent J. (31 March 2005). Malaysia car thieves steal finger. BBC Online. Kuala Lumpur. Archived from the original on 20 November 2010. Retrieved 11 December 2010.

31. Capgemini Research Institute. (2019) Reinventing Cybersecurity with Artificial Intelligence: The new frontier in digital security. Assessed from https://www.capgemini.com/wp-content/uploads/2019/07/AI-in-Cybersecurity_Report_20190711_V06.pdf

32. IBM Security. Cost of A Data Breach 2020. Assessed from https:// www.ibm.com/security/digital-assets/cost-data-breach-report/1Cost\%20of\%20a \%20Data\%20Breach\%20Report\%202020.pdf

33. NMER (June 2018). Economic and Financial Consequences of Corporate Cyberattacks. The Digest No. USA. Assessed from 
https://www.nber.org/digest/jun18/economic-and-financial-consequences-corporate-cyberattacks

34. Lease ML, Burke TW. Identity Theft: A Fast-Growing Crime. Journal FBI Law Enforcement Bulletin. August 2000;69(8): 8-13.

35. ITRC. 2021 Consumer Aftermath Report: How Identity Crimes Impact Victims, their Families, Friends, and Workplaces. Assessed from https:// www.idtheftcenter.org/publication/identity-theft-the-aftermath-study/

36. Lawson L. (January 21, 2022) 2021 Identity theft statistics. Trends and statistics about identity theft. Assessed from https://www.consumeraffairs.com/finance/identity-theft-statistics.html

37. Newman GR, McNally MM. (July 2005) Identity Theft Literature Review. Unpublished report of U.S. Department of Justice. Award Number: 2005TO-008. Document No.: 210459.

38. Benner J. Mierzwinski E, Givens B. (May 2000). Nowhere to turn: Victims speak out on identity theft. California Public Interest Research Group and the Privacy Rights Clearinghouse. Assessed from http://www.calpirg.org/consumer/privacy/idtheft2000/idtheft2000.pdf

39. Gordon GR, Curtis GE. (2000). The growing global threat of economic cyber crime. National Fraud Center, Inc. Assessed from http://www.lexisnexis.com/rissolutions/conference/docs/cyber.pdf

40. Insurance Information Institute. Facts + Statistics: Identity theft and cybercrime: The scope of identity theft. Assessed from https://www.iii.org/factstatistic/facts-statistics-identity-theft-and-cybercrime

41. Giact. U.S. Identity Theft: The Stark Reality. Assessed from https://giact.com/identity/us-identity-theft-the-stark-reality-report/

42. Bessen J. (February 17, 2022) How big technology systems are slowing innovation: The great IT revolution is no longer promoting economic dynamism. It's preventing it. Assessed from https://www.technologyreview.com/author/ james-bessen/

43. Mishra AK. Organizational responses to crisis the centrality of trust. In R.M. Kramer and T.R. Tyler (eds.). Trust in Organizations: Frontiers of Theory and Research. Thousand Oaks, CA: Sage, 1996. pp. 261-287. 
44. Chow S, Holden R. Toward an understanding of loyalty: The moderating role of trust. Journal of Managerial issue. 1997;9(3):275-298.

45. Weisbaum H. (July 30, 2018, Updated July 30, 2018). The total cost of a data breach - including lost business - keeps growing. Assessed from https:// www.nbcnews.com/business/consumer/total-cost-data-breach-including-lost-business-keeps-growing-n895826. 